\title{
Krankheitsbild Multiple Sklerose
}

Miriam Kip, Anne Zimmermann

1.1 Beschreibung und Einteilung nach der Verlaufsform - 2

1.2 Pathophysiologie und Ätiologie - 3

1.2.1 Pathophysiologie -3

1.2.2 Ätiologie -3

1.3 Krankheitszeichen und Krankheitsverlauf - 5

1.3.1 Frühe Krankheitszeichen und häufige Symptome -5

1.3.2 Krankheitsverlauf -7

1.4 Diagnostik und Behandlung - 8

Literatur -11 


\section{Zusammenfassung}

Die Multiple Sklerose ist die häufigste chronisch entzündlich-degenerative Erkrankung des Zentralen Nervensystems im jungen Erwachsenenalter. Charakteristisch sind fokale Demyelinisierungen und der Verlust von Nervenzellfasern sowie die zeitliche Dissemination der Läsionen. Die Ursachen der Erkrankung sind unklar. Es wird von einer multifaktoriellen, durch Umweltfaktoren getriggerten Autoimmunerkrankung im genetisch prädisponierten Menschen ausgegangen.

Zu den wahrscheinlichen Umweltfaktoren zählen u.a. eine Infektion mit dem Ebstein-Barr-Virus (im Kindesalter) oder Vitamin D-Mangel. Frauen erkranken im Vergleich zu Männern deutlich häufiger. Bei den meisten Patienten verläuft die Erkrankung in Schüben. Primär progrediente Verläufe, bei denen sich der Gesundheitszustand mit dem Krankheitsbeginn kontinuierlich verschlechtert, sind vergleichsweise selten. Das Beschwerdebild ist heterogen und schließt neuropsychologische Symptome wie Fatigue, Störungen der Kognition oder Depressionen, Spastik und Einschränkungen der Mobilität, Schmerzen, Störungen der Blasenfunktion sowie Störungen der Sexualität mit ein. Die Wahrscheinlichkeit bleibender Funktionseinschränkungen nimmt mit der Krankheitsdauer zu. Die individuellen Krankheitsverläufe sind aber sehr unterschiedlich und lassen sich nicht sicher voraussagen. Die Diagnosestellung erfolgt nach definierten klinischen und paraklinischen Kriterien (McDonald-Kriterien). Die Behandlung der Multiplen Sklerose besteht aus der verlaufsmodifizierenden Therapie (Schubprophylaxe) und der Therapie des akuten Schubes, die in der Stufentherapie zusammengefasst sind, sowie der symptomatischen Therapie. Die Erkrankung ist nicht heilbar. Die Stufentherapie umfasst Medikamente, die auf unterschiedliche Weisen das Immunsystem modulieren mit dem Ziel, das Fortschreiten der Erkrankung aufzuhalten. Die symptomatische Therapie besteht aus nicht-medikamentösen und medikamentösen Verfahren, die eine Linderung der Beschwerden anstreben.

\subsection{Beschreibung und Einteilung nach der Verlaufsform}

Die Multiple Sklerose (MS) ist eine chronisch entzündliche und degenerative Erkrankung des zentralen Nervensystems (ZNS).
In Abhängigkeit der Krankheitsaktivität und Progredienz der Erkrankung werden die folgenden Verlaufsformen der MS voneinander abgegrenzt (•Tab. 1.1, \ Kap. 4.1.2) (Lublin et al. 2014):

- Klinisch isoliertes Syndrom (KIS)

- Schubförmig verlaufende MS (Relapsing Remitting MS, RRMS)

- Sekundär progrediente MS (SPMS)

- Primär progrediente MS (PPMS)

Das radiologisch isolierte Syndrom (RIS) ist eine weitere Unterform der MS und beschreibt Patienten, bei denen in der Magnetresonanztomografie (MRT) zwar MS-typische Läsionen messbar, die Patienten aber klinisch asymptomatisch sind. Zwischen 30 und

- Tab. 1.1 ICD-10 GM Klassifikation der MS
ICD-10-Code

G35.0

G35.1

G35.10

G35.11

G35.2

G35.20

G35.21

G35.3

G35.30

G35.31

G35.9

\section{Erkrankung}

Erstmanifestation einer MS

MS mit vorherrschend schubförmigem Verlauf

MS mit vorherrschend schubförmigem Verlauf: Ohne Angabe einer akuten Exazerbation oder Progression MS mit vorherrschend schubförmigem Verlauf: Mit Angabe einer akuten Exazerbation oder Progression MS mit primär-chronischem Verlauf MS mit primär-chronischem Verlauf: Ohne Angabe einer akuten Exazerbation oder Progression

MS mit primär-chronischem Verlauf: Mit Angabe einer akuten Exazerbation oder Progression

MS mit sekundär-chronischem Verlauf

MS mit sekundär-chronischem Verlauf: Ohne Angabe einer akuten Exazerbation oder Progression

MS mit sekundär-chronischem Verlauf: Mit Angabe einer akuten Exazerbation oder Progression

MS, nicht näher bezeichnet
Quelle: IGES - DIMDI ICD-10 GM Version (2014) 
$45 \%$ dieser Patienten entwickeln einige Jahre später (im Median nach zwei bis fünf Jahren) typische Symptome einer RRMS oder SPMS (Lebrun 2015).

\subsection{Pathophysiologie und Ätiologie}

\subsubsection{Pathophysiologie}

Das pathologische Korrelat der MS sind multiple Entzündungsherde charakterisiert durch Entmarkungen (Demyelinisierungen) und Zerstörung von Nervenzellfasern im ZNS (axonale Destruktion und axonaler Verlust). Konfluierende Demyelinisierungen erscheinen als Plaques, die Degeneration und Verlust der Nervenzellfasern mündet in einer Hirnatrophie. Die Läsionen betreffen bevorzugt den Sehnerv, Hirnstamm, das Rückenmark, Kleinhirn und die die Gehirnventrikel umgebenden Areale (Wiendl u. Kieseier 2010). Die chronische Entzündungsreaktion, die die MS-typischen Läsionen im ZNS hervorrufen, und die Neurodegeneration, stehen in engem (zeitlichen) Zusammenhang (Dendrou et al. 2015; Garg u. Smith 2015).

Die Entzündungsreaktion ist am ehesten im Sinne einer Autoimmunreaktion zu verstehen, bei der Zellen des spezifischen (T-Lymphozyten, BLymphozyten) und unspezifischen Immunsystems (z.B. Makrophagen) die Blut-Hirn-Schranke überwinden und sich dort gegen das körpereigene, die Nervenfasern des ZNS (Oligodendrozyten) umhüllende, Myelin in Gehirn und Rückenmark wenden. Bei Verlust der Nervenscheiden (Demyelinisierung) verlangsamt sich die Erregungsleitung an den betroffenen Nerven, was der Patient beispielsweise als
Muskelschwäche oder Sensibilitätsstörungen wahrnimmt. Zu Beginn der Erkrankung ist der Körper imstande, Demyelinisierungen teilweise selbst durch Remyelinisierungen auszugleichen. Schädigungen und Verlust der Nervenfasern sind allerdings irreversibel und damit vor allen Dingen mit den bleibenden neurologischen Beeinträchtigungen der MS assoziiert (Dendrou et al. 2015; Garg u. Smith 2015). Die Entzündungsreaktion verläuft chronisch und ist bei allen Verlaufsformen der MS messbar. Sie ist besonders ausgeprägt in akuten Phasen der Erkrankung (bei der schubförmig verlaufenden MS) und nimmt mit Progredienz der Erkrankung ab. Die Degeneration tritt mit der Krankheitsdauer in den Vordergrund und ist gekennzeichnet durch Destruktion und Verlust der Nervenzellfasern und einer Atrophie des Hirngewebes. Degenerative Prozesse treten auch schon vor Krankheitsbeginn auf (subklinisch) (Dendrou et al. 2015; Garg u. Smith 2015) (• Abb. 1.1).

\subsection{2 Ätiologie}

Die Ätiologie (Ursachen) der MS ist unklar (Wiendl u. Kieseier 2010). Man geht davon aus, dass verschiedene Umweltfaktoren (•Tab. 1.2) in genetisch prädisponierten Menschen eine Störung in der Immunantwort auslösen, die die Demyelinisierungen und Degeneration der Nervenzellfasern hervorrufen (Dendrou et al. 2015, Garg und Smith 2015).

Hinweise auf einen Zusammenhang zwischen Infektionen mit verschiedenen Erregern und der MS wurden in verschiedenen Studien erbracht (Wiendl u. Kieseier 2010). Insbesondere eine Infek-

- Tab. 1.2 Mögliche Risiko- und Umweltfaktoren im Zusammenhang mit einer MS

Erhöhtes Risiko

Frauen

Genetische Prädisposition (HLA DR15/DQ6, IL2RA und IL7RA Allele)

\section{Umweltfaktoren}

Pathogene z.B. Ebstein-Barr Virus (EBV)

Wenig Sonnenexposition und Vitamin D-Mangel Rauchen

Übergewicht

Hohe Kochsalzzufuhr 


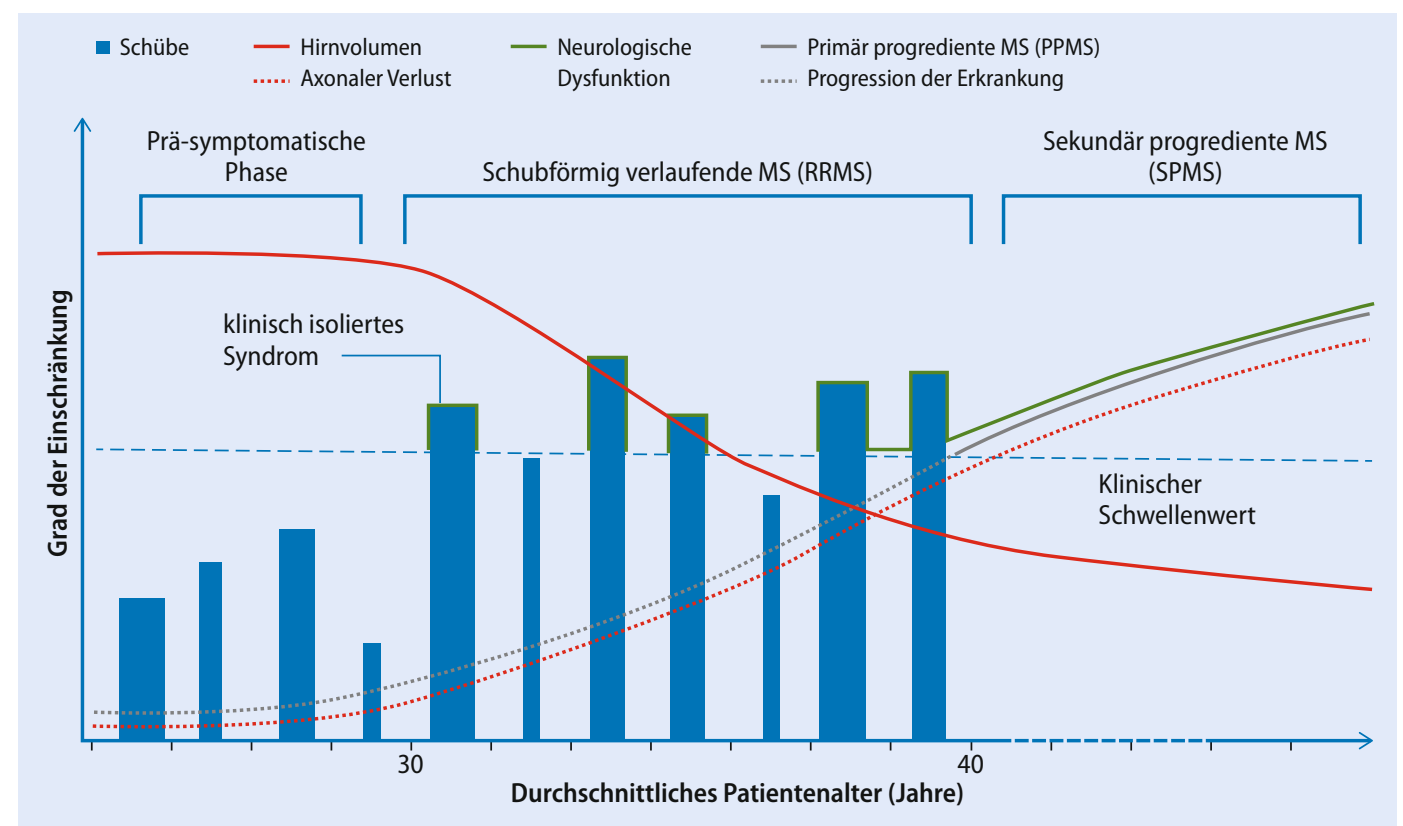

- Abb. 1.1 Zusammenhang von Entzündungs- und degenerativen Prozessen unter Berücksichtigung der Verlaufsformen der MS (Quelle: IGES - mit Genehmigung von Macmillan Publishers Ltd: Nature; modifiziert aus Dendrou et al. (2015))

tion mit dem Ebstein-Barr-Virus (EBV) ist mit einem erhöhten Risiko einer MS assoziiert. Wobei die Infektion allein die MS nicht auslöst, aber eine Funktionsstörung in der Immunantwort bei genetisch prädisponierten Menschen begünstigt, insbesondere dann, wenn die Infektion erst im Erwachsenenalter erfolgte (Garg u. Smith 2015; Wiendl u. Kieseier 2010).

Vor dem Hintergrund höherer Prävalenz- und Inzidenzangaben in Skandinavien im Vergleich zu südeuropäischen Ländern (Kingwell et al. 2013) wird seit einigen Jahren die Sonnenexposition und damit zusammenhängend Vitamin D als möglicher Umweltfaktor in der Entstehung und Verlauf der MS untersucht. Vitamin D wird im Körper insbesondere durch Sonneneinstrahlung gebildet und hat eine immunmodulatorische Wirkung (Wiendl $\mathrm{u}$. Kieseier 2010). Zum einen war höhere Sonnenexposition während der Kindheit und Jugend mit einem niedrigeren Risiko für MS verbunden, zum anderen war bei niedrigem Vitamin D-Level das Risiko, an einer MS zu erkranken, erhöht (Ascherio et al. 2014; Duan et al. 2014; Mokry et al. 2015). Derzeit wird in Studien untersucht, ob sich eine
Vitamin-D-Substitution positiv auf den Krankheitsverlauf auswirken kann (DMSG 2015a).

Auch die Ernährungsgewohnheiten unterscheiden sich regional, und so wird die hohe Kochsalzzufuhr über die Ernährung insbesondere in Industrieländern als ein weiterer Umweltfaktor im Zusammenhang mit der MS diskutiert (Hucke et al. 2015). Eine Assoziation zwischen Kochsalzzufuhr und MS-typischen Läsionen ist bislang im Tiermodell nachgewiesen. Neben einer immunmodulatorischen Wirkung (Kleinewietfeld et al. 2013), konnten Schädigungen der Blut-Hirn-Schranke bei hoher Kochsalzzufuhr gezeigt werden, was dazu führt, dass Immunzellen in das Gehirn migrieren können, was bei intakter Blut-Hirn-Schranke nicht möglich ist. Schädigungen der Blut-Hirn-Schranke waren geschlechtsspezifisch und traten bevorzugt bei Weibchen auf (Krementsov et al. 2015). Eine HDLcholesterinreiche Ernährung wiederum kann unter Umständen dazu beitragen, das Erkrankungsrisiko zu senken bzw. den Verlauf der Erkrankung günstig zu beeinflussen, da HDL-Cholesterin im Zusammenhang mit einer protektiven Wirkung auf die Blut-Hirn-Schranke diskutiert wird (Fellows et al. 
2015). Der genaue Einfluss der Ernährung auf den Verlauf der Erkrankung ist aber bisher unzureichend untersucht. Eine Korrelation der Darmflora (Mikrobiom) mit der Erkrankung ist wahrscheinlich. Die genauen Zusammenhänge und darauf basierend eine gezielte Modulation der Darmflora als mögliche Therapie der MS sind Gegenstand laufender Untersuchungen (DMSG 2015b).

Rauchen wurde ebenfalls als möglicher Risikofaktor für eine MS-Erkrankung identifiziert. Das relative Risiko, eine MS zu entwickeln, war in Studien bei Rauchern im Vergleich zu Nichtrauchern um das 1,5-Fache erhöht, wobei das Risiko mit zunehmender Dosis stieg (Wingerchuk 2012). Des Weiteren beeinflusst Rauchen die Krankheitsaktivität, und Patienten mit MS profitieren davon, mit dem Rauchen aufzuhören (Ramanujam et al. 2015).

Zudem zeigen sich in der Epidemiologie der MS geschlechtsspezifische Unterschiede. Frauen erkranken im Vergleich zu Männern deutlicher häufiger (ungefähr im Verhältnis 2-3:1) (Kingwell et al. 2013). In den letzten Jahrzehnten hat die Neuerkrankungsrate (Inzidenz) unter Frauen besonders stark zugenommen, wie internationale epidemiologische Studien zeigten (Koch-Henriksen u. Sorensen 2010; Westerlind et al. 2014a). Auch bei gleicher genetischer Prädisposition erkranken Frauen phänotypisch häufiger als Männer. Die Ursachen für die unterschiedliche Penetranz der Gene (Wahrscheinlichkeit, dass ein bestimmter Genotyp zu einem bestimmten Phänotyp führt) sind nicht geklärt (Wiendl u. Kieseier 2010), es wird aber diskutiert, ob eine unterschiedliche Exposition zu Umweltfaktoren eine Rolle spielt und in Teilen das erhöhte Erkrankungsrisiko von Frauen erklärt (Koch-Henriksen u. Sorensen 2010). Das Risiko, an einer MS zu erkranken, nimmt zwar mit dem Verwandtschaftsverhältnis bzw. mit dem Anteil gemeinsamen Genmaterials zu (Wiendl u. Kieseier 2010), das Vererbungsrisiko für die Nachkommen von MSErkrankten ist insgesamt aber nur gering erhöht (Westerlind et al. 2014b).

Die genetische Prädisposition hat nicht nur Einfluss auf das Erkrankungsrisiko, sondern bestimmt möglicherweise auch den Therapieverlauf mit. Es gibt Hinweise darauf, dass die therapeutische Wirksamkeit mancher Medikamente mit der Expression und Mutationen der Gene assoziiert ist, die auch mit der Erkrankung selbst in Zusammenhang stehen (Wiendl et al. 2010).

\subsection{Krankheitszeichen und Krankheitsverlauf}

\subsubsection{Frühe Krankheitszeichen und häufige Symptome}

Die Erkrankung manifestiert sich meist im jüngeren Erwachsenenalter durch ein oder mehrere Läsionen im Gehirn, Rückenmark oder am Sehnerv. Frauen im Alter von 20 bis 40 Jahre erkranken am häufigsten. In Abhängigkeit der betroffenen Areale im ZNS berichten die Patienten zu Krankheitsbeginn von einzelnen oder mehreren sensorischen, motorischen, visuellen oder Hirnstamm-Symptomen (Garg u. Smith 2015) sowie neurokognitiven und psychischen Beschwerden (Kister et al. 2013). Ein charakteristisches Symptom zu Krankheitsbeginn ist die Entzündung des Sehnervs (Optikusneuritis), begleitet von Doppelbildwahrnehmungen sowie Sehstörungen und Augenschmerzen. Weitere typische Symptome im frühen Krankheitsstadium sind Sensibilitätsstörungen wie Missempfindungen (Parästhesien), Taubheitsgefühle oder Schmerzen in den Extremitäten. Hinzu kommen Gangstörungen mit häufig belastungsabhängiger Schwäche der Beine sowie Gangunsicherheit. Jüngere Patienten leiden zunächst häufig unter einer Optikusneuritis als alleinigem Symptom oder Sensibilitätsstörungen. Bei älteren Patienten treten häufig zu Beginn Paresen als alleiniges Symptom oder in Kombination mit sensiblen Ausfällen auf (DGN 2014; Wiendl u. Kieseier 2010).

Aber auch neuropsychologische Symptome wie Fatigue oder kognitive Beeinträchtigungen treten früh im Krankheitsverlauf auf. Eine prospektive Verlaufsstudie unter Patienten aus den USA zeigte, dass Patienten sogar schon nach dem ersten demyelinisierenden Ereignis (KIS) und vor einer gesicherten Diagnose einer MS signifikant häufiger Symptome einer Fatigue zeigten im Vergleich zu gesunden Kontrollen. Das Ausmaß der Müdigkeitssymptomatik war unter den Patienten mit KIS vergleichbar der Symptomatik bei Patienten mit einer gesicherten MS und war ein unabhängiger Risiko- 
faktor für die spätere bestätigte Diagnose einer MS (Runia et al. 2015).

Die häufig mit einer MS assoziierten Symptome lassen sich grob in neuropsychologische Symptome (kognitive Beeinträchtigungen, Fatigue und Depression), motorische Einschränkungen und Einschränkungen der Mobilität (Spastik, Muskelschwäche, Ataxie und Tremor), Sensibilitätsstörungen und Schmerzen sowie Störungen der Blasen- und Darmfunktion sowie Störungen der Sexualität einteilen. Im Folgenden werden die häufigsten und am stärksten beeinträchtigenden Symptome beschrieben (DGN 2014).

Kognitive Beeinträchtigungen äußern sich unter anderem als Konzentrations- und Aufmerksamkeitsstörungen oder Abnahme von Gedächtnisleistungen. Sie haben eine starke Korrelation zu MS-typischen morphologischen Veränderungen im ZNS (Tiemann et al. 2009). Während bei milden bis moderaten Verläufen nicht betroffene Areale kognitive Funktionen übernehmen, nimmt die Fähigkeit der spontanen Rekrutierung neuronaler Systeme mit der Progression der Erkrankung ab (Penner et al. 2007).

Die Fatigue äußert sich in einer erhöhten Tagesmüdigkeit sowie muskulären und kognitiven Erschöpfung, die sich durch körperliche Anstrengung oder Wärme verschlechtert (Iriarte et al. 2000). Die primäre Fatigue wird als Folge der MS-spezifischen immunologischen Prozesse und neuroendokriner Fehlfunktion im Rahmen von Läsionen im ZNS erklärt (Patejdl et al. 2015). Eine sekundäre Fatigue liegt aufgrund indirekter Beschwerden vor, die wiederum eine Folge anderer MS-assoziierten Symptome sind, wie Blasenfunktionsstörungen, Depression oder gestörte Nachtruhe bei Schmerzen. Auch andere Erkrankungen wie eine Anämie oder Unterfunktion der Schilddrüse können die Ermüdungssymptomatik zusätzlich verschlimmern (Patejdl et al. 2015).

Die Depression zählt ebenfalls zu den häufigen und sehr beeinträchtigenden Begleitsymptomen in allen Phasen der Erkrankung (Feinstein et al. 2014). Eine depressive Symptomatik ist unter anderem mit der Fatigue assoziiert und begünstigt eine schlechte Adhärenz bezüglich der verlaufsmodifizierenden Therapie (Tarrants et al. 2011).

Motorische Symptome und Einschränkungen der Mobilität aufgrund von Symptomen wie Spastik,
Muskelschwäche, Ataxie und Tremor führen ebenfalls $\mathrm{zu}$ starken funktionellen Beeinträchtigungen. Die Spastik beschreibt eine Zunahme des Muskeltonus mit Reduktion der Kraft und Ausdauerleistung des Muskels. Sie kann intermittierend oder kontinuierlich bestehen und von Schmerzen begleitet sein. Die Ataxie ist ein Begriff für die gestörte Bewegungskoordination, die insbesondere das Gehen oder Stehen betrifft. Sie betrifft auch die oberen Extremitäten und ist häufig bei einer zielgerichteten Bewegung, wie beim Greifen eines Glases, von Zittern (Intentionstremor) begleitet (DMSG 2004). Weitere Symptome können die Augenmotorik, das Schlucken (Dysphagie) und Sprechen (Dysarthrie) betreffen.

Schmerzen kommen nicht nur im Zusammenhang mit der Spastik vor. Es werden verschiedene Arten des Schmerzes unterschieden, die bei einer MS-Erkrankung häufig in Kombination auftreten, entsprechend ihrer Genese aber unter Umständen unterschiedlich therapiert werden (DGN 2015; DMSG 2004). Der mit einer MS am häufigsten auftauchende Schmerz ist der neuropathische Schmerz, welcher die Unterbrechung oder Veränderung von Nervenleitungen zur Ursache hat und somit eine direkte Folge der MS-Erkrankung beschreibt. Patienten berichten häufig über Kopfschmerzen, Missempfindungen, Brennen (Dysästhesien) oder Neuralgien.

Blasenstörungen äußern sich beispielsweise in Form von Inkontinenz, Harnverhalt (bei Spastik) oder Drangsymptomen. Als Folge werden Harnwegsinfekte und Schädigungen der Nieren begünstigt (DGN 2014). Darmfunktionsstörungen treten als Obstipation oder Inkontinenz auf (DMSG 2004).

Der Erkrankungsbeginn der MS liegt meist zwischen dem 20. und 40. Lebensjahr und betrifft somit den Lebensabschnitt, in welchem neben der beruflichen Karriere Partnerschaften gebildet und Familien geplant werden. Störungen der Sexualität sind daher häufig nicht nur ein individuelles, sondern auch ein partnerschaftliches Problem. Neben den neurologischen Ursachen kommen des Weiteren die signifikant erhöhten Symptome von Depression und Angst als Ursache in Frage. Zudem haben der Umgang mit der Erkrankung und die Bewältigungsstrategien innerhalb einer partnerschaftlichen Beziehung einen wesentlichen Einfluss auf die sexuelle Funktionalität und Aktivität. Es besteht die Gefahr, 
- Tab. 1.3 Auswahl häufiger Symptome unter Patienten mit MS auf Basis des Datensatzes der DMSG $(n=16.554)$ in Abhängigkeit von der Krankheitsdauer

\begin{tabular}{|c|c|c|}
\hline Symptom & $\begin{array}{l}\text { Krankheitsdauer } \\
<2 \text { Jahre (\%) }\end{array}$ & $\begin{array}{l}\text { Krankheitsdauer } \\
>15 \text { Jahre (\%) }\end{array}$ \\
\hline \multicolumn{3}{|l|}{ Neuropsychologisch } \\
\hline Fatigue & 40,6 & 67,6 \\
\hline Kognitive Einschränkungen & 19,6 & 40,6 \\
\hline Depressionen & 23,6 & 38,0 \\
\hline \multicolumn{3}{|l|}{ Motorisch } \\
\hline Spastiken & 17,3 & 75,8 \\
\hline Ataxie/Tremor & 24,4 & 56,5 \\
\hline Schmerzen & 24,6 & 42,2 \\
\hline Blasenstörungen & 20,5 & 74,0 \\
\hline Darmstörungen & 4,8 & 31,1 \\
\hline Sexuelle Dysfunktion & 8,0 & 27,3 \\
\hline Andere & 2,1 & 3,9 \\
\hline $\begin{array}{l}\text { Quelle: IGES - Stuke et al. (2008) } \\
\text { Anmerkung: DMSG = Deutsche } \mathrm{N}\end{array}$ & & \\
\hline
\end{tabular}

dass sich das Gleichwertigkeitsgefühl des Betroffenen mindert. Zudem kann es ihm aufgrund der fluktuierenden Symptome schwer fallen, sich dem Partnern gegenüber zu erklären, was wiederum zur Folge haben kann, dass die Probleme als psychische Leiden interpretiert werden (Beier et al. 2002; Goecker et al. 2006).

\subsubsection{Krankheitsverlauf}

Die klinische Symptomatik ist aufgrund der für die MS typischen multiplen und oft unterschiedlichen Lokalisierung der Läsionen im ZNS äußerst heterogen und auch der Schweregrad der Symptome unterliegt einer großen Variationsbreite. Bleiben die Symptome länger als sechs Monate bestehen, sinkt ihre Rückbildungswahrscheinlichkeit und die Häufigkeit der mit der Erkrankung assoziierten Symptome und bleibenden Einschränkungen nimmt mit der Krankheitsdauer zu (• Tab. 1.3) (Kister et al. 2013; Stuke et al. 2008).
Insbesondere die Anzahl der Patienten mit Spastiken, motorischen Einschränkungen, Ataxien/Tremore sowie Blasen- und Darmfunktionsstörungen nimmt mit der Krankheitsdauer zu, wie eine Auswertung des North American Registry of Multiple Sclerosis (NARCOMS Kohorte) zeigte. NARCOMS beinhaltet Selbstausauskünfte zu Krankheitszeichen, Krankheitsverlauf und Therapien von über 35.000 Patienten mit MS aus dem Zeitraum 1996 bis 2011. Dabei wurde die Erkrankungshäufigkeit einzelner Symptome bzw. Symptomkomplexe im Zusammenhang mit der Erkrankungsdauer (von weniger als ein Jahr bis 30 Jahre) betrachtet.

Mit der Krankheitsdauer verschlimmerte sich auch der Schweregrad bleibender körperlicher Einschränkungen (Kister et al. 2013). Laut DMSG war nach einer durchschnittlichen Krankheitsdauer von 12,7 Jahren ( $\pm 9,2$ Jahre) noch gut die Hälfte (51 \%) der Patienten des DMSG-Datensatzes uneingeschränkt gehfähig und wies geringfügige Einschränkungen auf, $28 \%$ benötigten eine Gehilfe, um 100 m 
laufen zu können und $6 \%$ waren bei schwer eingeschränkter Gehfähigkeit an einen Rollstuhl gebunden (Flachenecker et al. 2008).

Neuropsychologische Symptome nehmen ebenfalls an Häufigkeit und Schweregrad mit der Krankheitsdauer zu. Die Häufigkeit dieser Symptome scheint aber im Vergleich zur Spastik, Einschränkungen der Mobilität oder den Blasen- wie Darmfunktionsstörungen weniger deutlich mit der Krankheitsdauer assoziiert zu sein (Kister et al. 2013).

Die (bleibenden) neurologischen Ausfälle und neurokognitiven Einschränkungen können in einer eingeschränkten Erwerbsfähigkeit oder Frühberentung resultieren. Dies ist besonders schwerwiegend, denn die meisten Patienten erkranken jung in einer Lebensphase der beruflichen Findung und Etablierung. Nur 27,9 \% der im Datensatz der DMSG dokumentierten Patienten waren voll berufstätig, $39,4 \%$ bezogen Rentenleistungen aufgrund von Berufs- und Erwerbsunfähigkeit, 6 \% waren arbeitslos. Mit zunehmenden Grad der Behinderung steigt die Anzahl der Patienten im vorzeitigen Ruhestand (Flachenecker et al. 2008).

Der individuelle Krankheitsverlauf lässt sich bei Krankheitsbeginn nicht sicher voraussagen. Es sind aber einige Faktoren (demografische, klinische und paraklinische) identifiziert worden, die mit einem erhöhten Risiko eines ungünstigen Krankheitsverlaufs und den Folgen der Erkrankung assoziiert sind
(- Tab. 1.4) (DGN 2014). Auch bei Patienten mit RRMS unter Therapie werden unterschiedliche Verläufe in Abhängigkeit des Behinderungsgrades beobachtet, wobei der Zeitpunkt des Übergangs von milden zu schweren Verläufen auch hier nicht sicher vorausgesagt werden kann (Scott et al. 2014).

\subsection{Diagnostik und Behandlung}

Das diagnostische Vorgehen bei Patienten mit Verdacht auf das Vorliegen einer MS hat das Ziel einer zügigen und sicheren Bestätigung der Diagnose, indem die klinischen Symptome erfasst, objektiviert und quantifiziert werden. Die Diagnosestellung der MS erfolgt nach definierten Kriterien (McDonaldKriterien), die schwerpunktmäßig auf klinischen und MS-typischen Veränderungen in bildgebenden Verfahren (MRT) basieren. Die Liquoruntersuchung und elektrophysiologische Verfahren (evozierte Potenziale) können zusätzlich hinzugezogen werden. Die Liquordiagnostik ist allerdings nur in Ausnahmefällen für die Diagnosestellung der MS selbst relevant, sondern dient hauptsächlich der differenzialdiagnostischen Abklärung ( $\downarrow$ Kap. 3) (DGN 2014). Insbesondere bei nur einem Krankheitszeichen oder mehreren atypischen Symptomen bei der Erstkonsultation ist eine Abgrenzung zu anderen entzündlichen, z.B. erregerbedingten Erkran-

- Tab. 1.4 Übersicht relevanter Einflussfaktoren auf die Progression

Günstiger Verlauf

Erkrankungsbeginn $<35$. Lebensjahr

Schubförmiger Verlauf

Geringe Schubrate mit großen Abständen zwischen Schüben und guter Rückbildungstendenz

Monosymptomatischer Beginn: sensible Symptome oder Sehnerventzündung

Geringe Behinderung in früher Krankheitsphase

Unauffälliges MRT zu Beginn

Keine pathologischen SEP und MEP
Ungünstiger Verlauf

Erkrankungsbeginn $>50$. Lebensjahr

Progredienter Verlauf

Hohe Schubrate mit geringen Abständen zwischen Schüben und schlechter Rückbildungstendenz

Polysymptomatischer Beginn: motorische und zerebelläre Symptome

Hoher Behinderungsgrad schon in ersten Krankheitsjahren

Zahlreiche und große Läsionen in MRT

Früh pathologische SEP und MEP 
- Tab. 1.5 Sonderformen

\begin{tabular}{|l|l|}
\hline ICD-10-Code & Erkrankung \\
\hline G36.0 & $\begin{array}{l}\text { Neuromyelitis optica (NMO; } \\
\text { Devic Syndrom) }\end{array}$ \\
\hline G04.0 & $\begin{array}{l}\text { Akut disseminierte/demyelinisie- } \\
\text { rende Enzephalomyelitis (ADE) }\end{array}$ \\
\hline G37.5 & $\begin{array}{l}\text { Balos konzentrische Sklerose (Ence- } \\
\text { phalomyelitis periaxialis concentrica) }\end{array}$ \\
\hline G37.0 & $\begin{array}{l}\text { Diffus disseminierte Sklerose (Schil- } \\
\text { der-Krankheit; Encephalitis periaxia- } \\
\text { lis diffusa) }\end{array}$ \\
\hline
\end{tabular}

Quelle: IGES - Wiendl u. Kieseier (2010); DIMDI ICD-10 GM (Version 2014)

kungen oder anderen demyelinisierenden Erkrankungen des ZNS und Sonderformen der MS notwendig (•Tab. 1.5) (DGN 2014; Wiendl u. Kieseier 2010).

Wegen der Notwendigkeit eines frühzeitigen Beginns der medikamentösen, verlaufsmodifizierenden Therapie (Schubprophylaxe) und der Prävention bleibender Einschränkungen hat die frühe, sichere Diagnosestellung einen hohen Stellenwert.

Auch wenn die MS bislang nicht heilbar ist, stehen im Jahr 2016 eine Vielzahl therapeutischer Optionen zur Verfügung, mittels derer die Erkrankung behandelbar ist (Ransohoff et al. 2015). Die Behandlung von Patienten mit MS setzt sich aus der verlaufsmodifizierenden Therapie und der Therapie des akuten Schubs (Stufentherapie) ( $\triangleright$ Abschn. 4.1) und der symptomatischen Therapie ( $\triangleright$ Abschn. 4.2) zusammen (DGN 2014). Die verlaufsmodifizierende Therapie hat das Ziel, die Krankheitsaktivität medikamentös einzudämmen (no evidence of disease activity, NEDA), Schübe zu verhindern und damit den Krankheitsverlauf zu bremsen. Ziel in der Behandlung der RRMS ist eine an die Krankheitsaktivität angepasste Therapie (DGN 2014).

Verallgemeinernd gesprochen wirken alle für die verlaufsmodifizierende Therapie zugelassenen Medikamente als Immunmodulatoren, die mehr oder weniger spezifisch das Immunsystem beeinflussen (Garg u. Smith 2015; Wiendl u. Meuth 2015). Bei der Auswahl der Therapie wird der indi- viduelle Nutzen (Effektivität) gegenüber den Risiken abgewogen (Havla et al. 2015; Ransohoff et al. 2015). Die Therapie ist dann effektiv, wenn die klinische und paraklinische Krankheitsaktivität reduziert ist. Ziel ist - wie oben beschrieben - ein $\mathrm{Zu}$ stand frei von Krankheitsaktivität (keine Schübe, keine neue Läsionen im MRT) (DGN 2014). Die Risiken der verlaufsmodifizierenden Therapie sind medikamentenspezifisch. Sie können unter anderem Nebenwirkungen wie Infektionen, progressive multifokale Leukenzephalopathie (PML) oder Neoplasien umfassen (• Tab. 1.6) (DGN 2014; Garg u. Smith 2015).

Mit Zunahme der Progredienz nehmen die therapeutischen Möglichkeiten aber ab. Für die primär progrediente Verlaufsform sind aktuell formal keine Medikamente zugelassen (DGN 2014). Medikamente, die primär darauf abzielen, bestehende Schädigungen am Nerven zu reparieren, wie beispielsweise verschiedene Remyelinisierungstherapien sind noch Gegenstand von Untersuchungen (Harlow et al. 2015) ( Kap. 6).

Ziel der symptomatischen Therapie ist eine Reduktion bzw. Stabilisierung von Funktionseinschränkungen sowie die Vermeidung von symptombedingten Komplikationen und die Verbesserung der Lebensqualität. Kern der symptomatischen Behandlung ist die Rehabilitation. Die Behandlung beinhaltet nicht-medikamentöse Maßnahmen wie z. B. Physiotherapie und neuropsychologische Therapien gegebenenfalls im Rahmen der multimodalen (stationären) Rehabilitation und medikamentöse Verfahren.

Aus Perspektive der Patienten stehen die Minderung der Symptome und das Vorbeugen einer Progression der Erkrankung im Vordergrund der Behandlung. Patienten sind bereit, ein Risiko von schweren Nebenwirkungen oder Tod in Höhe von einem Prozent hinzunehmen, wenn dafür das Fortschreiten der Symptome für zehn Jahre oder die Progression für sieben Jahre verhindert wird. Das gleiche Risiko waren Patienten bereit, für eine orale Therapie aufzunehmen oder für eine Therapie, welche die Symptome selten, aber dafür erheblich bessert (Wilson et al. 2015). 
- Tab. 1.6 Wichtige Nebenwirkungen zugelassener Therapien für die verlaufsmodifizierende Therapie der MS nach Garg u. Smith (2015)

\begin{tabular}{|c|c|}
\hline Arzneimittel* & Häufige Nebenwirkungen \\
\hline Alemtuzumab & $\begin{array}{l}\text { Schwere Infusionsreaktionen, sekundäre autoimmune Schilddrüsenerkrankung, Thrombo- } \\
\text { zytopenie, Erkrankung der Anti-Glomerulären Basalmembran, erhöhtes Risiko für maligne } \\
\text { Schilddrüsenkarzinome, Melanome, lymphoproliferative Störungen }\end{array}$ \\
\hline Interferon Beta-1a & $\begin{array}{l}\text { Grippesymptome, Reaktionen an der Injektionsstelle, Erhöhung der Leberenzymwerte, } \\
\text { Schilddrüsen-Anomalien, Leukopenie oder Anämie, Depressionen }\end{array}$ \\
\hline Interferon Beta-1b & $\begin{array}{l}\text { Grippesymptome, Reaktionen an der Injektionsstelle, Erhöhung der Leberenzymwerte, } \\
\text { Schilddrüsen-Anomalien, Leukopenie oder Anämie, Depressionen }\end{array}$ \\
\hline Dimethylfumarat & $\begin{array}{l}\text { Gastrointestinale Beschwerden wie Übelkeit, Diarrhö oder abdominale Schmerzen; Hitze- } \\
\text { wallungen, Juckreiz, Erhöhung der Leberenzymwerte, Lymphopenie, seltene Fälle von PML }\end{array}$ \\
\hline Fingolimod & $\begin{array}{l}\text { Nach erstmaliger Einnahme Bradykardie, AV-Block, Herpes-Virus Infektionen, Makulaödem, } \\
\text { erhöhter Blutdruck, geringes Risiko von PML }\end{array}$ \\
\hline Glatirameracetat & $\begin{array}{l}\text { Reaktionen an der Injektionsstelle, Folgereaktionen nach Injektion (Hitzewallung, Engegefühl } \\
\text { im Brustkorb, Herzklopfen, Atemnot), selten Lipoatrophie bei dauerhafter Verabreichung }\end{array}$ \\
\hline Mitoxantron & Kardiotoxizität, sekundäre Leukämie \\
\hline Natalizumab & Lebertoxizität, Infusionsreaktionen, PML \\
\hline PEG-Interferon Beta-1a & $\begin{array}{l}\text { Grippesymptome, Reaktionen an der Injektionsstelle, Erhöhung der Leberenzymwerte, } \\
\text { Schilddrüsen-Anomalien, Leukopenie oder Anämie, Depressionen }\end{array}$ \\
\hline Teriflunomid & $\begin{array}{l}\text { Haarausfall, Kopfschmerzen, Diarrhö, Hepatotoxizität, Teratogenität, erhöhtes Risiko für } \\
\text { Infektionen aufgrund von Lymphopenie }\end{array}$ \\
\hline
\end{tabular}

Open Access Dieses Kapitel wird unter der Creative Commons Namensnennung-Nicht kommerziell 4.0 International Lizenz (http://creativecommons.org/licenses/by-nc/4.0/deed.de) veröffentlicht, welche für nicht kommerzielle Zwecke die Nutzung, Vervielfältigung, Bearbeitung, Verbreitung und Wiedergabe in jeglichem Medium und Format erlaubt, sofern Sie den/die ursprünglichen Autor(en) und die Quelle ordnungsgemäß nennen, ein Link zur Creative Commons Lizenz beifügen und angeben, ob Änderungen vorgenommen wurden.

Etwaige Abbildungen oder sonstiges Drittmaterial unterliegen ebenfalls der genannten Creative Commons Lizenz, sofern sich aus der Abbildungslegende oder der Quellreferenz nichts anderes ergibt. Sofern solches Drittmaterial nicht unter der genannten Creative Commons Lizenz steht, ist eine Vervielfältigung, Bearbeitung oder öffentliche Wiedergabe nur mit vorheriger Zustimmung des betreffenden Rechteinhabers oder auf der Grundlage einschlägiger gesetzlicher Erlaubnisvorschriften zulässig. 


\section{Literatur}

Ascherio A, Munger KL, White R, Kochert K, Simon KC, Polman $\mathrm{CH}$, Freedman MS, Hartung HP, Miller DH, Montalban X, Edan G, Barkhof F, Pleimes D, Radu EW, Sandbrink R, Kappos L, Pohl C (2014) Vitamin D as an early predictor of multiple sclerosis activity and progression. JAMA Neurol 71(3), 306-314. DOI: 10.1001/jamaneurol.2013.5993.

Beier KM, Goecker D, Babinsky S, Ahlers CJ (2002) Sexualität und Partnerschaft bei Multipler Sklerose - Ergebnisse einer empirischen Studie bei Betroffenen und ihren Partnern. Sexuologie 9(1), 4-22.

Dendrou CA, Fugger L, Friese MA (2015) Immunopathology of multiple sclerosis. Nat Rev Immunol 15(9), 545-558. DOI: 10.1038/nri3871.

DGN (Hrsg.) (2014) Leitlinien für Diagnostik und Therapie in der Neurologie. Diagnose und Therapie der Multiplen Sklerose. Entwicklungsstufe: S2e. Stand: Januar 2012, Ergänzung August 2014. Gültig bis 2017. (AWMF-Registernummer: 030/050). Deutsche Gesellschaft für Neurologie. http://www.awmf.org/uploads/tx_szleitlinien/ 030-050l_S2e_Multiple_Sklerose_Diagnostik_Therapie_2014-08_verlaengert.pdf [Abruf am: 04.11.2015].

DGN (Hrsg.) (2015): Leitlinien für Diagnostik und Therapie in der Neurologie. Pharmakologisch nicht interventionelle Therapie chronisch neuropathischer Schmerzen. Entwicklungsstufe: S1. Veröffentlicht September 2012, Ergänzt 7.1.2014, Gültig bis 31. Dezember 2016. (AWMF-Registernummer: 030/114). Berlin: Deutsche Gesellschaft für Neurologie. http://www.awmf.org/ uploads/tx_szleitlinien/030-114I_S1_Neuropathischer_ Schmerzen_Therapie_2014-01.pdf [Abruf am: 18. Dezember 2015].

DMSG (2004) Symptomatische Therapie der Multiplen Sklerose - Aktuelle Therapieempfehlungen Multiple Sklerose Therapie Konsensus Gruppe (MSTKG). Hannover: Deutsche Multiple Sklerose Gesellschaft Bundesverband e.V.

DMSG (2015a) Kanadische Studie belegt: Genetischer Vitamin-D-Mangel erhöht das Multiple Sklerose-Risiko. Letzte Änderung: 26.08.2015. Hannover: Deutsche Multiple Sklerose Gesellschaft Bundesverband e.V. http://www. dmsg.de/multiple-sklerose-news/index. php?w3pid=news\&kategorie $=$ forschung\&anr $=5700 \&-$ suchbegriffe=vitamin\%20d [Abruf am 04. Januar 2016].

DMSG (2015b) Multiple Sklerose: Wie Darmbakterien die Gesundheit des Gehirns beeinflussen. Letzte Aktualisierung: 24. September 2015. Hannover: Deutsche Multiple Sklerose Gesellschaft Bundesverband e.V. http:// www.dmsg.de/multiple-sklerose-news/index. php?w3pid=news\&kategorie $=$ forschung\&anr $=5727 \&$ suchbegriffe=Ern\%E4hrung [Abruf am: 04. Januar 2016].

Duan S, Lv Z, Fan X, Wang L, Han F, Wang H, Bi S (2014) Vitamin $D$ status and the risk of multiple sclerosis: a systematic review and meta-analysis. Neurosci Lett 570, 108-113. DOI: 10.1016/j.neulet.2014.04.021.

Feinstein A, Magalhaes S, Richard JF, Audet B, Moore C (2014) The link between multiple sclerosis and depression. Nat
Rev Neurol 10(9), 507-517. DOI: 10.1038/nrneurol. 2014.139.

Fellows K, Uher T, Browne RW, Weinstock-Guttman B, Horakova D, Posova H, Vaneckova M, Seidl Z, Krasensky J, Tyblova M, Havrdova E, Zivadinov R, Ramanathan M (2015) Protective associations of HDL with blood-brain barrier injury in multiple sclerosis patients. J Lipid Res. 56(10), 2010-2018. DOI: 10.1194/jlr.M060970.

Flachenecker P, Stuke K, Elias W, Freidel M, Haas J, PitschnauMichel D, Schimrigk S, Zettl UK, Rieckmann P (2008) Multiple sclerosis registry in Germany: results of the extension phase 2005/2006. Deutsches Ärzteblatt 105(7), 113-119. DOI: 10.3238/arztebl.2008.0113.

Garg N, Smith TW (2015) An update on immunopathogenesis, diagnosis, and treatment of multiple sclerosis. Brain Behav 5(9), e00362. DOI: 10.1002/brb3.362.

Goecker D, Rösing D, Beier KM (2006) Der Einfluss neurologischer Erkrankungen auf Partnerschaft und Sexualität: Unter besonderer Berücksichtigung der Multiplen Sklerose und des Morbus Parkinson. Der Urologe 45(8), 992-998. DOI: 10.1007/s00120-006-1094-7.

Harlow DE, Honce JM, Miravalle AA (2015) Remyelination Therapy in Multiple Sclerosis. Front Neurol 6, 257. DOI: 10.3389/fneur.2015.00257.

Havla J, Kumpfel T \& Hohlfeld R (2015): [Immunotherapies for multiple sclerosis : review and update]. Internist (Berl) 56(4), 432-445. DOI: 10.1007/s00108-015-3668-1.

Hucke S, Wiendl H, Klotz L (2015) Implications of dietary salt intake for multiple sclerosis pathogenesis. Mult Scler Oct 7. DOI: $10.1177 / 1352458515609431$.

Iriarte J, Subira ML, Castro P (2000) Modalities of fatigue in multiple sclerosis: correlation with clinical and biological factors. Mult Scler 6(2), 124-130. ISSN: 1352-4585 (Print), 1352-4585 (Linking).

Kingwell E, Marriott JJ, Jette N, Pringsheim T, Makhani N, Morrow SA, Fisk JD, Evans C, Beland SG, Kulaga S, Dykeman J, Wolfson C, Koch MW, Marrie RA (2013) Incidence and prevalence of multiple sclerosis in Europe: a systematic review. BMC Neurol 13, 128. DOI: 10.1186/1471-237713-128.

Kister I, Bacon TE, Chamot E, Salter AR, Cutter GR, Kalina JT, Herbert J (2013) Natural history of multiple sclerosis symptoms. Int J MS Care 15(3), 146-158. DOI: 10.7224/ 1537-2073.2012-053.

Kleinewietfeld M, Manzel A, Titze J, Kvakan H, Yosef N, Linker RA, Muller DN, Hafler DA (2013) Sodium chloride drives autoimmune disease by the induction of pathogenic TH17 cells. Nature 496(7446), 518-522. DOI: 10.1038/ nature11868.

Koch-Henriksen N, Sorensen PS (2010) The changing demographic pattern of multiple sclerosis epidemiology. Lancet Neurol 9(5), 520-532.

Krementsov DN, Case LK, Hickey WF, Teuscher C (2015) Exacerbation of autoimmune neuroinflammation by dietary sodium is genetically controlled and sex specific. FASEB J 29(8), 3446-3457. DOI: 10.1096/fj. 15-272542. 
Lebrun C (2015) The radiologically isolated syndrome. Rev Neurol (Paris) 171(10), 698-706. DOI: 10.1016/j.neurol. 2015.05.001.

Lublin FD, Reingold SC, Cohen JA, Cutter GR, Sorensen PS, Thompson AJ, Wolinsky JS, Balcer LJ, Banwell B, Barkhof $F$, Bebo B, Jr., Calabresi PA, Clanet M, Comi G, Fox RJ, Freedman MS, Goodman AD, Inglese M, Kappos L, Kieseier BC, Lincoln JA, Lubetzki C, Miller AE, Montalban X, O'Connor PW, Petkau J, Pozzilli C, Rudick RA, Sormani MP, Stuve O, Waubant E, Polman CH (2014) Defining the clinical course of multiple sclerosis: the 2013 revisions. Neurology 83(3), 278-286. DOI: 10.1212/WNL.0000000000000560.

Mokry LE, Ross S, Ahmad OS, Forgetta V, Smith GD, Leong A, Greenwood CM, Thanassoulis G, Richards JB (2015) Vitamin D and Risk of Multiple Sclerosis: A Mendelian Randomization Study. PLoS Med 12(8), e1001866. DOI: 10.1371/journal.pmed.1001866.

Patejdl R, Penner IK, Noack TK, Zettl UK (2015) [Fatigue in patients with multiple sclerosis--pathogenesis, clinical picture, diagnosis and treatment]. Fortschr Neurol Psychiatr 83(4), 211-220. DOI: 10.1055/s-0034-1399353.

Penner IK, Opwis K, Kappos L (2007) Relation between functional brain imaging, cognitive impairment and cognitive rehabilitation in patients with multiple sclerosis. Journal of Neurology \& Neurophysiology 254 Suppl 2, II53-57. DOI: 10.1007/s00415-007-2013-6.

Ramanujam R, Hedstrom AK, Manouchehrinia A, Alfredsson L, Olsson T, Bottai M, Hillert J (2015) Effect of Smoking Cessation on Multiple Sclerosis Prognosis. JAMA Neurol 72(10), 1117-1123. DOI: 10.1001/jamaneurol.2015.1788.

Ransohoff RM, Hafler DA, Lucchinetti CF (2015) Multiple sclerosis-a quiet revolution. Nat Rev Neurol 11(3), 134142. DOI: 10.1038/nrneurol.2015.14.

Runia TF, Jafari N, Siepman DA, Hintzen RQ (2015) Fatigue at time of CIS is an independent predictor of a subsequent diagnosis of multiple sclerosis. J Neurol Neurosurg Psychiatry 86(5), 543-546. DOI: 10.1136/jnnp-2014308374.

Scott TF, Hackett CT, Quigley MR, Schramke CJ (2014) Relapsing multiple sclerosis patients treated with disease modifying therapy exhibit highly variable disease progression: a predictive model. Clin Neurol Neurosurg 127, 86-92. DOI: 10.1016/j.clineuro.2014.09.008.

Stuke K, Flachenecker P, Zettl U, Elias W, Freidel M, Haas J, Pitschnau-Michel D, Schimrigk S, Rieckmann P (2008) MS-Register in Deutschland 2008 - Symptomatik der MS. http://www.dmsg.de/dokumentearchiv/dgn2008_ msregister.pdf [Abruf am: 19. Mai 2015].

Tarrants M, Oleen-Burkey M, Castelli-Haley J \& Lage MJ (2011) The impact of comorbid depression on adherence to therapy for multiple sclerosis. Multiple sclerosis international 2011, 271321. DOI: 10.1155/2011/271321.

Tiemann L, Penner IK, Haupts M, Schlegel U, Calabrese P (2009) Cognitive decline in multiple sclerosis: impact of topographic lesion distribution on differential cognitive deficit patterns. Mult Scler 15(10), 1164-1174. DOI: $10.1177 / 1352458509106853$.
Westerlind H, Bostrom I, Stawiarz L, Landtblom AM, Almqvist C, Hillert J (2014a) New data identify an increasing sex ratio of multiple sclerosis in Sweden. Mult Scler 20(12), 1578-1583. DOI: 10.1177/1352458514530021.

Westerlind H, Ramanujam R, Uvehag D, Kuja-Halkola R, Boman M, Bottai M, Lichtenstein P, Hillert J (2014b) Modest familial risks for multiple sclerosis: a registry-based study of the population of Sweden. Brain 137(Pt 3), 770-778. DOI: 10.1093/brain/awt356.

Wiendl H, Kieseier BC (2010) Multiple Sklerose. Klinik, Diagnostik und Therapie. Klinische Neurologie. Stuttgart: Kohlhammer. ISBN: 978-3170184633.

Wiendl H, Meuth SG (2015) Pharmacological Approaches to Delaying Disability Progression in Patients with Multiple Sclerosis. Drugs 75(9), 947-977. DOI: 10.1007/s40265-0150411-0.

Wilson LS, Loucks A, Gipson G, Zhong L, Bui C, Miller E, Owen M, Pelletier D, Goodin D, Waubant E, McCulloch CE (2015) Patient preferences for attributes of multiple sclerosis disease-modifying therapies: development and results of a ratings-based conjoint analysis. Int J MS Care 17(2), 74-82. DOI: 10.7224/1537-2073.2013-053.

Wingerchuk DM (2012) Smoking: effects on multiple sclerosis susceptibility and disease progression. Therapeutic advances in neurological disorders 5(1), 13-22. DOI: $10.1177 / 1756285611425694$. 